\title{
ASSESSMENT OF THE BIODIVERSITY OF SAMPLES USED FOR ISOLATION OF MICROBIAL STRAINS CAPABLE OF CONVERTING STRAW DESTINED AS A SUBSTRATE FOR BIOGAS PLANT
}

\author{
Krystyna Cybulska' ${ }^{1}$ Paweł Kołosowski², Ilona Wrońska' ${ }^{1}$, Tomasz Dobek² \\ 1 Department of Microbiology and Biotechnology of Enviroment, West Pomeranian University of Technology \\ in Szczecin, Słowackiego 17, 71-434 Szczecin, Poland, e-mail: krystyna.cybulska@zut.edu.pl, ilona.wronska@ \\ zut.edu.pl \\ 2 Department of Construction and Use of Technical Devices, West Pomeranian University of Technology in \\ Szczecin, Papieża Pawła VI/3, 71-459 Szczecin, Poland, e-mail: pawel.kolosowski@zut.edu.pl, tomasz.dobek@ \\ zut.edu.pl
}

Received: 2015.11.16

Accepted: 2015.12.09

Published: 2016.01 .06

\begin{abstract}
In biogas plants, almost any type of organic matter can be used as a substrate to produce biogas. To make the process of methane fermentation more effective, these materials are pretreated. This applies in particular to a group of difficult substrates. Straw, due to its hemicellulose structure and saturation, is hardly fermented by biogas reactor microorganisms. The methods of post-harvest residue preparation for anaerobic digestion being applied so far are expensive, while their application has a negative effect on methanoegenic bacteria. Therefore, the microorganisms being able to degrade straw hemicellulose structure, utilisation of which could precede the proper fermentation process, have been searched for. This paper presents the results of microbial biodiversity analysis in the environmental samples being lupin, cereal, rape and maize straw as well as hay and haylage at different degradation stages. The analysis of biodiversity will help at a further stage of study to isolate active microbial strains showing cellulolytic, hemicellulolytic or ligninolytic activity which are desirable in the process of straw biodegradation. Analysis of the microbial count was performed by the method of deep inoculation on different microbiological culture media. The conducted tests include determination of the number of fungi, bacteria and actinomycetes. The results obtained confirm the usefulness of the analysed samples for isolation of microbial strains capable of converting straw preceding the biogas production.
\end{abstract}

Keywords: straw, biological preteatment, isolation of microorganisms strains, biogas plant, enzymatic activity.

\section{INTRODUCTION}

Despite the large potential of straw as a substrate for biogas production, its use is marginal at present. This is mainly due to the structure of materials of that type, where cellulose, hemicellulose and lignin prevail [Mussato and Teixeira, 2004; Adapa et al., 2009; Chaturvedi and Verma, 2013]. Such a structure prevents the effective use of straw by biogas reactor microflora [Frei, 2013]. Great hopes are placed on the possibility of using microorganisms in the straw pretreatment [Dorado et al, 1999; Feng et al., 2012].
Properly selected microbial species would allow effective straw processing at relatively low cost [Devi and Kumar, 2012; Zakpaa et al., 2010]. A potential habitat of useful microbes is straw stockpiles, where the material being considerably degraded is to be found. Properly selected microorganisms could solve the problem, which would allow that substrate to be used at a large scale [Taherzedeh and Karimi, 2008]. Different groups of microorganisms can live in the straw undergoing natural process of biodegradation. This applies to both fungi and bacteria and actinomycetes [Ashraf et al., 2007; Nagaraju et 
al.,2009]. A substantial group of these microorganisms have the ability to secrete cellulolytic enzymes [Huang et al., 2014].

This study aimed at assessing the microbial biodiversity of selected straw materials showing signs of biodegradation, understood as an analysis of the number of bacteria, fungi and actinomycetes inhabiting the examined samples.

\section{MATERIAL AND METHODS}

The samples intended for biodiversity assessment were collected on straw material stockpiles being stored for a period of 1 to 4 years, showing different degree of decomposition. The study analysed degraded blue lupin and winter rape straws, hay, basic cereal straw, corn straw, haylage and intact barley straw. A series of dilutions was prepared from the samples collected, which were used for making microbiological cultures by deep inoculation. Inoculations were made using selective microbiological culture media allowing a wide spectrum of microorganisms inhabiting the analysed material, including bacteria, actinomycetes and fungi, to grow. To grow actinomycetes, a Ciganov (CG) medium was used [Cyganow and Zukov, 1964]. In order to grow bacteria, an MPA culture medium (BTL Co. Ltd, Poland) was used, as well as a modified Bunt and Rovira (BR) medium [Bunt and Rovira, 1955], in which the soil extract was replaced with barley straw. The fungi culture was conducted on the following culture media: Martin (MR - Rose Bengal Agar) medium [Martin, 1950], Potato Dextrose Agar (PDA) medium (Oxoid Ltd) and Czapek Dox Agar (CDA) medium (BioCorp Co. Ltd, Poland). Two mineral culture media, containing grounded straw as the only source of carbon, were also used. The first one was Mandel agar medium (MAM) [Mandels and Weber, 1969], used for growing the fungi showing cellulolytic activity, whereas the second a modified Omelianski's medium $(\mathrm{OM})$ (with the following composition per $1: 1 \mathrm{~g}$ $\mathrm{KNO}_{3}, 1 \mathrm{~g} \mathrm{~K}_{2} \mathrm{HPO}_{4}, 0.5 \mathrm{~g} \mathrm{MgSO}_{4} \cdot 7 \mathrm{H}_{2} \mathrm{O}$, traces of $\mathrm{NaCl}, 10 \mathrm{~g}$ barley straw, $20 \mathrm{~g}$ agar). The cultures were conducted at a room temperature for a period of 3 to 7 days.

After incubation, the number of colony forming units (CFU) of a given group of microorganisms was determined per gram of sample's dry matter. One-way analysis of variance was performed with Statistica 10 software package. Using the Duncan's test, homogenous groups were distinguished from the samples under analysis. Homogenous groups were emerged among the same medium.

\section{RESULTS AND DISCUSSION}

The findings show that bacteria were the most numerous group of microorganisms inhabiting the samples under analysis. When making inoculation of two different growth media (BR medium and MPA medium), different data referring to their numbers were obtained, depending on the type of straw (Figure 1). On both media, a significant amount of bacteria was observed in the sample containing lupine straw and fresh barley straw. Based on the numbers of bacteria obtained on BR medium, particular straw materials were included into two homogenous groups.

Significantly lower numbers were observed for cereal and rape straws and hay. The average number of bacteria in this group was $3.43 \cdot 10^{8}$. On the other hand, 5-fold higher numbers were observed for lupine, corn and barley straws and haylage. In the case of MPA medium, the difference between the most represented sample containing haylage and the poorest one being a degraded cereal straw was almost 10 -fold. Like on BR medium, cereal straw and hay constituted a group with the lowest number of bacteria. The average number of bacteria in these materials was $8.31 \cdot 10^{8}$. Interestingly, rape straw characterised in the previous culture variant by one of the lowest numbers of bacteria was distinguished by their significantly higher number. The highest numbers of bacteria, $2.05 \cdot 10^{9} \mathrm{CFU} \cdot \mathrm{g}^{-1}$ D.M., were observed on MPA medium in the sample containing haylage.

The next group of microorganisms, the number of which had been analysed, was actinomycetes. Their determinations were made on Ciganov medium (Figure 1). Actinomycetes proved to be less numerous when compared bacteria. The highest number of actinomycetes was observed in lupine straw. A difference between lupine straw and other samples was significantly higher. By comparison, in the sample not showing any signs of degradation, i.e. in fresh barley straw, no growth of that group of microorganisms was observed. 


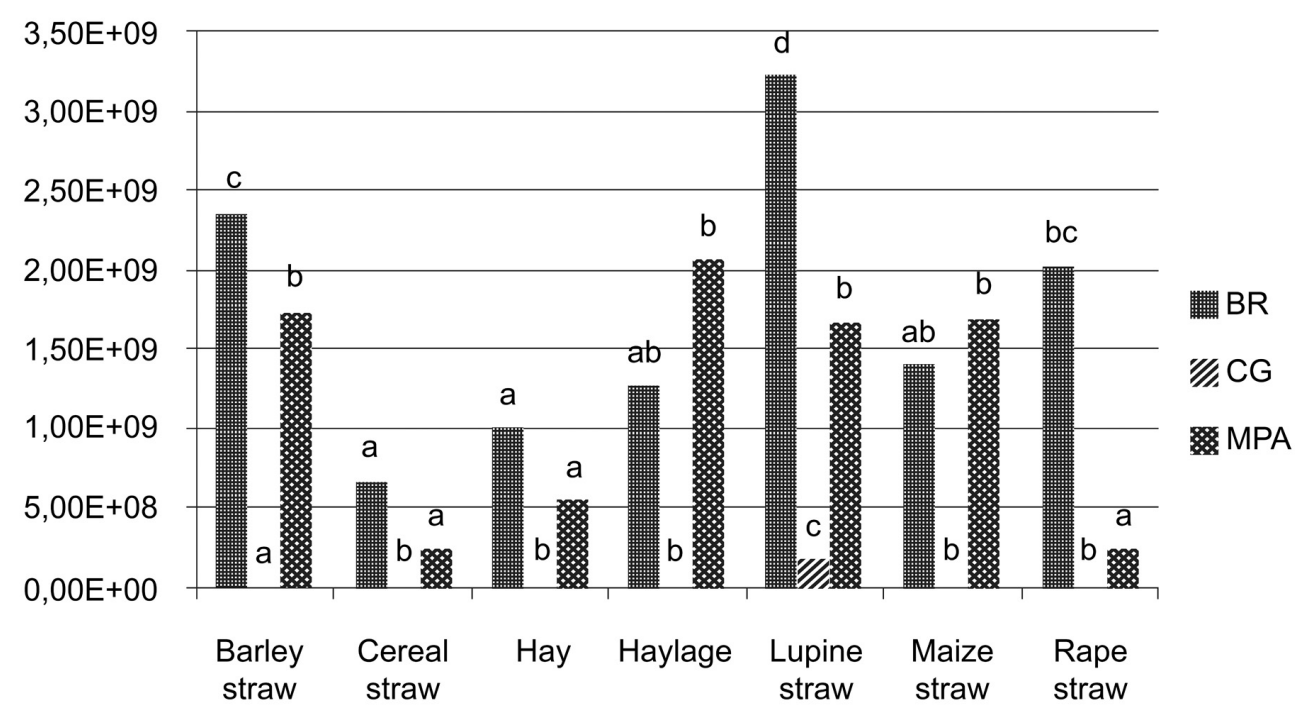

Figure 1. Number of bacteria and actinomycetes (CFU.g-1 D.M.) determined on different culture medium

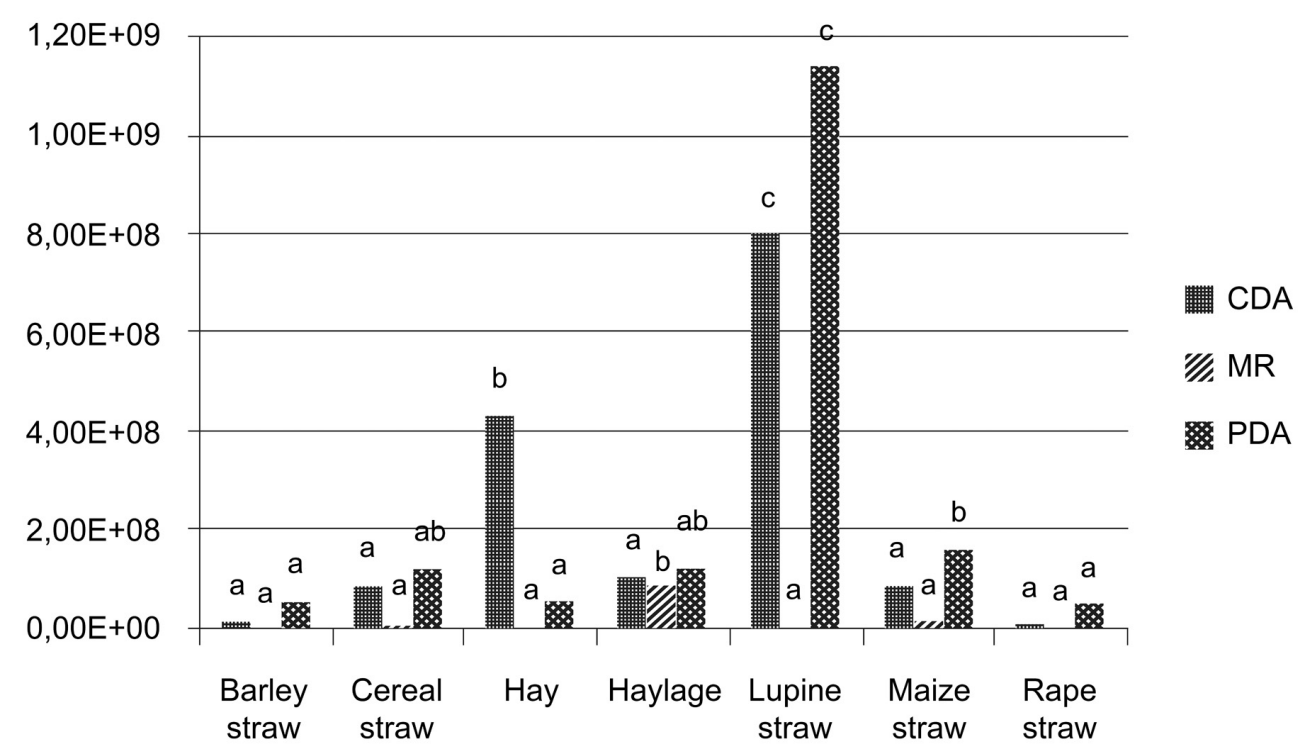

Figure 2. Number of fungi (CFU.g-1 D.M.) determined on different culture medium

Fungi biodiversity was analysed on three culture media: PDA medium, CDA medium and MR medium (Figure 2). The highest amount of them was observed on PDA medium, irrespective of the sample type. When analysing the number of fungi that grew on PDA medium, a clearly visible dominance of their number in lupine straw is observed when compared with other samples. In lupine straw, about $1.14 \cdot 10^{9} \mathrm{CFU} \cdot \mathrm{g}^{-1}$ D.M. was observed. Other samples were characterised by significantly lower numbers of fungi. On average, a 20-fold lower number of fungi was observed in rape and barley straws and hay, whereas this number was 6 times lower in cereal and corn straws and haylage.

The next growth medium for fungi being used in the experiment was CDA culture medium. Also in this case, similarly to PDA medium, fungi were observed most frequently in degraded cereal straw. Different results were obtained on MR medium. After statistical analysis of the results for the number of fungi, 2 homogenous groups were distinguished. One group included haylage only, which absolutely dominated over the rest of samples in terms of the number of fungi colony forming units being observed, whereas all other straw materials under analysis were assigned to the second one. A small number of fungi grown from lupine straw on this medium is worth mentioning.

Considering the research objective, the most important group of isolated microorganisms was those being able to use straw as a source of carbon and energy. The cultures of such microorgan- 


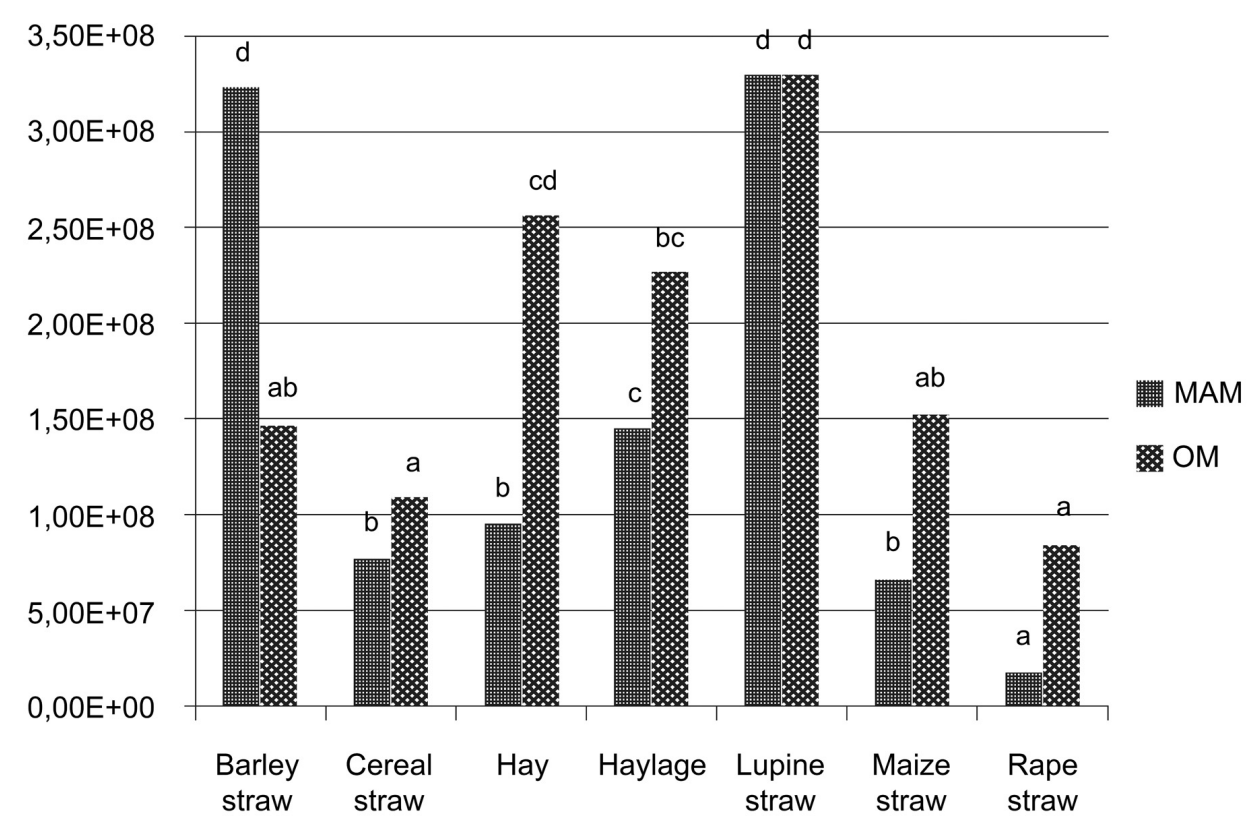

Figure 3. Number of cellulolytic microorganisms (CFU·g-1 D.M.) determined on different culture medium

isms were conducted on Mandel and Omelianski media, where powdered barley straw was applied as the only source of organic carbon. On OM medium, development of different species of microorganisms was observed, including bacteria, fungi and actinomycetes (Figure 3 ).

On the other hand, mainly fungi were cultured on MAM medium, as expected. After the analysis of the results of inoculation on OM medium, it is possible to state that the highest number of microorganisms being able to decompose straw was obtained in the sample containing lupine straw. A slightly lower number of straw-using microorganisms was obtained in hay. The least numerous group was rape straw, degraded cereal straw, fresh barley straw and corn straw. The next culture medium, on which the microorganisms being able to decompose straw had been grown, was Mandel medium. The least number of these micro-organisms was observed in rape straw. This material was inhabited by $1.91 \cdot 10^{7} \mathrm{CFU} \cdot \mathrm{g}^{-1}$ D.M. microorganisms being able to decompose straw. This was 17 times lower than in barley straw and lupine straw.

Fungi, in addition to bacteria, play an important role in biodegradation of biomass. Fungi are a group of microorganisms showing different demands towards the substrate being present in culture medium. When comparing the results obtained on PDA medium with the number of bacterial colonies obtained on BR medium, a ratio of 1.5:10 was received. However, due to high metabolic activity of the fungal mycoflora in- habiting composts, their participation in biomass decomposition cannot be overestimated and may amount even to 47 to $70 \%$ [Wiegant, 1991]. Decomposition of organic matter is associated with development of actinomycetes. However, their development seems to depend more on the availability of water and mineral substances than in case of bacteria and fungi. This may be indicated by the lack of activity of actinomycetes in undegraded barley straw. About $6 \%$ of actinomycetes in the sample with lupine straw is similar to the result being obtained by Ashiraf et al. [2007] who, when analysing the biodiversity of microorganisms in composters, receive a value of $8 \%$. In the process of straw conversion, preceding its processing in biogas production plant, only the strains of microorganisms being able to use this substrate as a source of carbon or energy can be applied. The inoculation on Mendel and Omelianski culture mediums, containing powdered straw, attempted to distinguish such species. The growth on these culture media requires the ability to excrete cellulase. In the materials subjected to composting, many species of prokaryotic organisms (9\%) [Yasurin et al., 2013] and fungi (46\%) show the cellulolytic activity. By comparing the number of microorganisms that grew on Omelanski medium with the total of colony forming units being calculated based on the analysis of bacteria inoculation on BR medium, fungi inoculation on PDA medium and actinomycete inoculation on Ciganov medium, it is possible to deduce that barely $9.5 \%$ of microflora inhabiting 
the samples under analysis is able to decompose straw. On the other hand, the result of comparison of the number of fungi that grew on Mandel medium (being able to decompose straw) with their number on PDA medium, which is $61 \%$, is very interesting.

\section{CONCLUSIONS}

Different types of straw materials, such as lupine straw, rape straw, hay, basic cereal straw, corn straw, fresh barley straw and haylage, being at different stage of decomposition, are numerously inhabited by bacteria, fungi and actinomycetes. Culture media for bacteria (BR and MPA mediums), culture media for fungi (PDA, Czapek and Martin mediums), growth medium for actinomycetes (Cignanov medium) and mineral culture media supplemented with powdered straw (Omelanski and Mandel mediums) allow assessment of the biodiversity of materials being examined. Lupine straw was most numerously inhabited by bacteria, fungi and actinomycetes; rape straw was the poorest in terms of the number of bacteria, whereas fresh barley straw was inhabited the least by fungi and actinomycetes. The highest number of microorganisms being potentially able to decompose straw on mineral culture media, where straw constituted a main source of carbon, was observed in lupine straw.

\section{REFERENCES}

1. Adapa P., Tabil L., Schoenau G. 2009. Compaction characteristics of barley, canola, oat and wheat straw. Biosyst. Eng., 104(3), 335-344. DOI: 10.1016/j.biosystemseng.2009.06.022.

2. Ashraf R., Shahid F., Ali T.A. 2007. Association of fungi, bacteria and actynomycetes with different compost. Pak. J. Bot. 39(6), 2141-2151.

3. Bunt J.S., Rovira A.D. 1955. Microbiological studies of some subantarctic soil. J. Soil Sci. 6(1), 119-128.

4. Chaturvedi V., Verma P. 2013. An overview of key pretreatment processes employed for bioconversion of lignocellulosic biomass into biofuels and value added products. 3 Biotech. 3, 415-431. DOI: 10.1007/s13205-013-0167-8.

5. Cyganow W.A., Zukov R.A. 1964. Morfologobiochimiciskije osobiennosti novowoviale actionomicita. Mikrobiologija 33(5), 863-869.
6. Devi M.C., Kumar M.S. 2012. Isolation and screening of lignocellulose hydrolytic saprophytic fungi from dairy manure soil. Annals of Biological Research 3(2), 1145-1152.

7. Doradoa J., Almendros G., Camarero S., Martinez A.T., Vares T., Hatakka A. 1999. Transformation of wheat straw in the course of solid-state fermentation by four ligninolytic basidiomycetes. Enzyme and Microbial Technology 25, 605-612.

8. Feng Y., Zhao X., Guo Y., Yang G., Xi J., Ren G. 2012. Changes in the material characteristics of maize straw during the pretreatment process of methanation. Journal of Biomedicine and Biotechnology 2012, 1-7. DOI: 10.1155/2012/325426.

9. Frei M. 2013. Lignin: characterization of a multifaceted crop component. The Scientific World Journal 2013, 1-25. DOI: 10.1155/2013/436517.

10. Huang G.L., Anderson T.D., Clubb R.T. 2014. Engineering microbial surfaces to degrade lignocellulosic biomass. Bioengineered. 5(2), 96-106.

11. Mandels M., Weber J. 1969. Production of cellulases. Adv. Chem. Ser. 95, 391-414.

12. Martin J.P. 1950. Use of acid, rose-bengal and streptomycin in the plate method for estimating soil fungi. Soil Sci. 69, 215-232.

13. Mussato S.I., Teixeira J.A. 2010. Lignocellulose as raw material in fermentation processes. Current Research, Technology and Education Topics in Applied Microbiology and Microbial Biotechnology 2, 897-907.

14. Nagaraju M., Narasihma G., Rangaswamy V. 2009. Impact of sugar industry effluents on soil cellulase activity. International Biodeterioration \& Biodegradation 63, 1088-1092. DOI: 10.1016/j. ibiod.2009.09.006.

15. Taherzadeh M.J., Karimi K. 2008. Pretreatment of lignocellulosic wastes to improve ethanol and biogas production: A review. Int. J. Mol. Sci. 9, 1621-1651. DOI: 10.3390/ijms9091621.

16. Wiegant W.M. 1991. A simple method to estimate the biomass of thermophilic fungi in composts. Biotechnol. Tech. 5(6), 421-426.

17. Yasurin P., Saenghiruna T., Phetsom J., Sriariyanun M. 2013. A study of feasibility to utilize lignocellulosic biomass as materials for biodiesel production. Official Conference Proceedings. The Official Conference Proceedings of The Asian Conference on Sustainability, Energy and the Environment 2013. Osaka. Japan, 686-693.

18. Zakpaa H.D., Mak-Mensah E.E., Johnson F.S. 2010. Saccharification of maize agrowastes by cellulolytic fungi isolated from Ejura Farms in Ejura, Ghana. Journal of Science and Technology 30(1), 10-17. 